\title{
Thyrotoxicosis after COVID-19 vaccination: seven case reports and a literature review
}

\author{
Kyung Ae Lee ${ }^{1} \cdot$ Yu Ji Kim ${ }^{1}$ Heung Yong Jin $\mathbb{1}^{1}$
}

Received: 22 September 2021 / Accepted: 30 September 2021 / Published online: 12 October 2021

(C) The Author(s), under exclusive licence to Springer Science+Business Media, LLC, part of Springer Nature 2021

\section{Dear Editor,}

Since the emergence of the new coronavirus disease-2019 (COVID-19) pandemic, several vaccines have been approved. As vaccination progresses, several cases indicating a possible relationship between thyroid diseases and vaccination have been reported. Here, we describe seven independent patients who visited Jeonbuk National University Hospital from March 2021 to July 2021, all presenting with thyrotoxicosis after COVID-19 vaccination. The underlying etiologies were identified through diagnostic workup, with three cases of Graves' disease (GD), two cases of subacute painful thyroiditis (SAT), one case of concurrent GD and SAT, and one case of painless thyroiditis (PT). Patient 1.1 was a nurse and had normal thyroid function before the vaccine. Patients 1.2 and 1.3 also had normal thyroid function performed at our hospital before vaccinations. The other patients did not undergo thyroid function test before vaccination although had no symptoms. After vaccination, symptoms, including neck pain or weakness in the lower extremities, were judged to be caused by the vaccine. The laboratory and imaging results of all seven cases are shown in Table 1. The changes in free thyroxine levels after treatment are shown in Fig. 1. To the best of our knowledge, this is the first report of thyroid diseases that developed following COVID-19 vaccination in Korea. The case of co-occurrence of SAT and GD and the case of PT are the first reports in the English literature related to the COVID-19 vaccine.

The illness, which involves mild flu-like forms to severe multi-organ failure and death in some patients, caused by severe acute respiratory syndrome coronavirus

Heung Yong Jin

mdjinhy@jbnu.ac.kr

1 Division of Endocrinology and Metabolism, Department of Internal Medicine, Research Institute of Clinical Medicine of Jeonbuk National University-Biomedical Research Institute of Jeonbuk National University Hospital, Jeonbuk National University Medical School, Jeonju, South Korea
2 (SARS-CoV-2) has been a threat since its emergence in late 2019. As of August 2021, more than 200 million individuals worldwide and more than 4 million individuals in Korea have been affected by COVID-19. Several types of vaccines, such as mRNA, adenovirus vectored, and inactivated, have been licensed in different countries. Various vaccines produce immunity through different mechanisms. Initial reviews of vaccination reported a satisfactorily high level of safety and protection against the disease. However, since the start of vaccination, several cases of thyroid dysfunction have been reported. To date, eight and four cases of SAT and newly diagnosed or recurrent GD, respectively, have been reported in the literature [1-7]. Most of the reported cases occurred after mRNA vaccination although were also caused by adenoviral vector vaccine and inactivated vaccine. Two mechanisms have been suggested regarding post-vaccination thyroid dysfunction. Autoimmune/inflammatory syndrome induced by vaccine adjuvants (ASIA) was first suggested in 2011 [8]. Adjuvants in vaccines are used as immunogenicity enhancing agents to induce the adaptive immune response and trigger adverse immune reactions causing ASIA syndrome. Previously, type 1 diabetes mellitus, primary ovarian failure, adrenal insufficiency, and thyroiditis (mostly SAT) have been reported to be related to ASIA syndrome after human papillomavirus, hepatitis B virus, and influenza vaccination [9]. Another mechanism is an autoimmune response after COVID-19 vaccination due to the spike glycoprotein of SARS-CoV-2 sharing a genetic similarity with a human protein [10].

Conversely, all three diseases reported in the present case report are major etiologies of thyrotoxicosis. SAT is a wellknown inflammatory disorder of the thyroid presenting with painful thyroid swelling and destructive thyrotoxicosis and is believed to be linked to upper respiratory viral infection or a post-viral inflammatory process. PT is suggested to be a part of the spectrum of autoimmune thyroid (AIT) diseases. GD is one of the AIT diseases caused by autoantibodies binding to the thyroid stimulating hormone receptor antibody (TSHR-Ab), stimulating the thyroid, and 


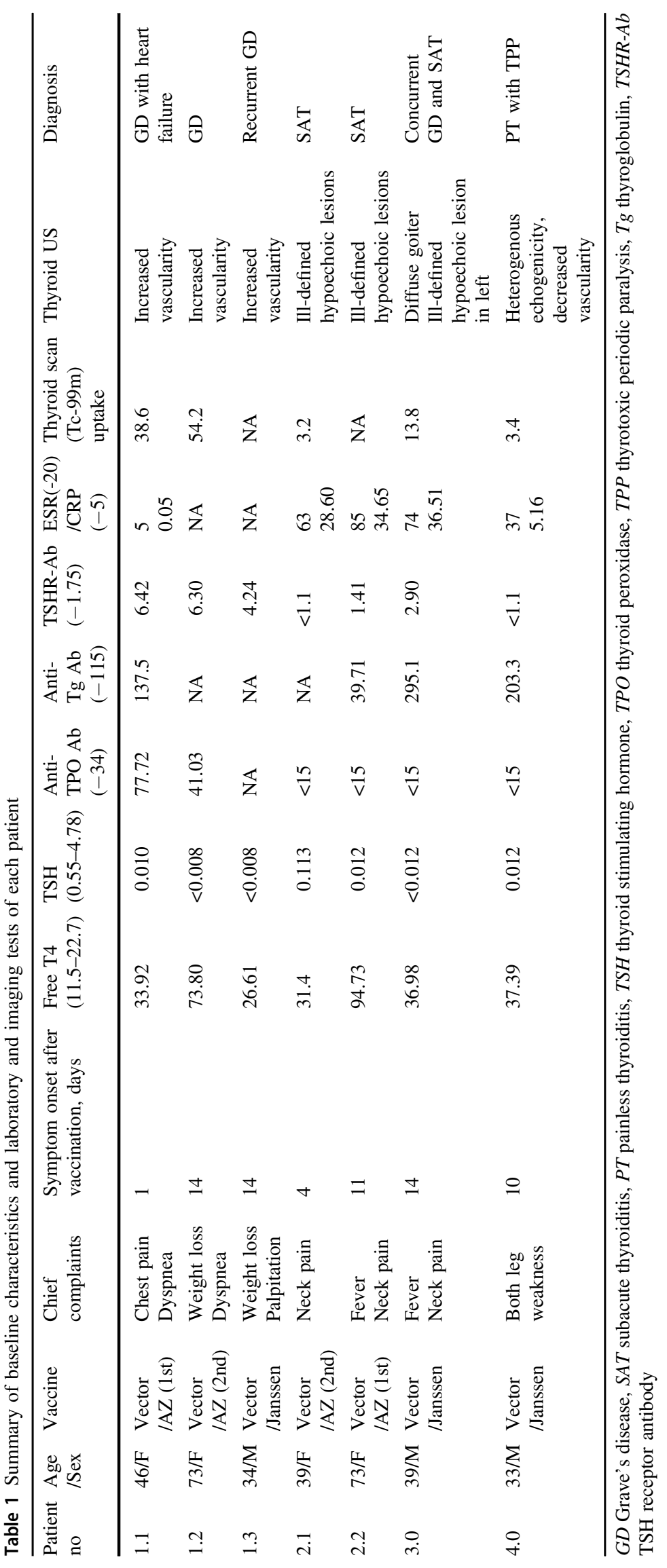




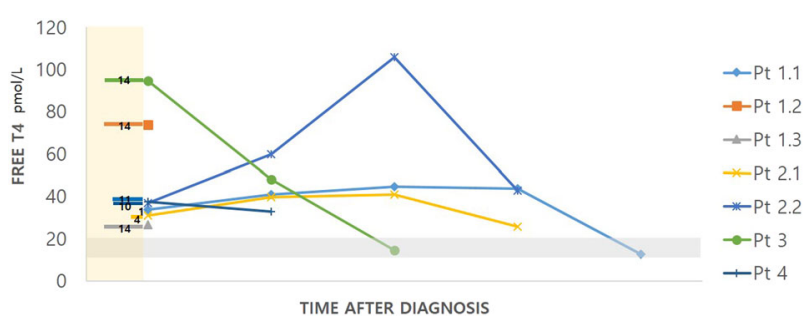

Fig. 1 Time to symptom onset after COVID-19 vaccination and free thyroxine (T4) change after diagnosis. The number in the yellow box represents the time from vaccination to symptom onset for each patient. The mean duration from vaccination to symptom onset was 9.7 (1-14) days. The gray box indicates the normal range of free T4

overproducing thyroid hormones. Regardless of the similar symptoms and signs of thyrotoxicosis, a differential diagnosis is important because the clinical course and treatment differ depending on the cause. Except for neck pain observed in SAT, it is difficult to differentiate based on symptoms alone. In some patients, more than one causal disease may occur sequentially or simultaneously. In particular, GD following SAT is not uncommon; SAT may promote the development of autoimmune diseases by promoting TSHR-Ab and subsequent release of thyroid antigens. GD concurrent with SAT is very rare but can occur, with only a few cases reported in the literature.

Based on case reports, including present cases and literature reviews, COVID-19 vaccines can cause not only destructive thyroiditis but also AIT. Clinical caution is needed because undiagnosed or delayed diagnosis of thyrotoxicosis can lead to the development of a fatal crisis. Furthermore, studies are warranted to clarify the relationship between COVID-19, vaccines, and thyroid diseases.

\section{Compliance with ethical standards}

Conflict of interest The authors declare no competing interests.
Ethical approval This study was approved by the Institutional Review Board (IRB) of Jeonbuk National University Hospital, and the requirement for informed consent was waived (IRB No. 2021-08-040).

Publisher's note Springer Nature remains neutral with regard to jurisdictional claims in published maps and institutional affiliations.

\section{References}

1. B.G. Iremli, S.N. Sendur, U. Unluturk, Three cases of subacute thyroiditis following SARS-CoV-2 vaccine: post-vaccination ASIA Syndrome. J Clin Endocrinol Metab. 106(9), 2600-2605 (2021)

2. S.O. Oyibo, Subacute thyroiditis after receiving the adenovirusvectored vaccine for Coronavirus Disease (COVID-19). Cureus. 13(6), e16045 (2021)

3. T.M. Sahin, S. Saylisoy, G. Yorulmaz, Subacute thyroiditis following COVID-19 vaccination in a 67-year-old male patient: a case report. Hum Vaccin Immunother 1, 1-3 (2021)

4. O. Vera-Lastra, N.A. Ordinola, M.P. Cruz, G. Medina, T.I. Sanchez, L.J. Jara, Two cases of Graves' disease following SARSCoV-2 vaccination: An autoimmune/inflammatory syndrome induced by adjuvants. Thyroid 31(9), 1436-1439 (2021)

5. G. Zettinig, M. Krebs, Two further cases of Graves' disease following SARS-Cov-2 vaccination. J Endocrinol Investig. 3, 1-2 (2021)

6. J. Schimmel, E.L. Alba, A. Chen, M. Russell, R. Srinath, Letter to the editor: thyroiditis and thyrotoxicosis after the SARS-CoV-2 mRNA vaccine. Thyroid 31(9), 1440 (2021)

7. C. Bornemann, K. Woyk, C. Bouter, Case report: two cases of subacute thyroidtisi following SARS-CoV-2 vaccination. Front Med. 24(8), 737142 (2021)

8. Y. Shoenfeld, N. Agmon-Levin, 'ASIA' - autoimmune/inflammatory syndrome induced by adjuvants. J Autoimmun. 36(1), 4-8 (2011)

9. N.L. Bragazzi, A. Hejly, A. Watad, M. Adawi, H. Amital, Y. Shoenfeld, ASIA syndrome and endocrine autoimmune disorders. Best Pract Res Clin Endocrinol Metab. 34(1), 101412 (2020)

10. W. Chen, Y. Tian, Z. Li, J. Zhu, T. Wei, J. Lei, Potential interaction between SARS-CoV-2 and thyroid: a review. Endocrinology 162(3), bqab004 (2021) 